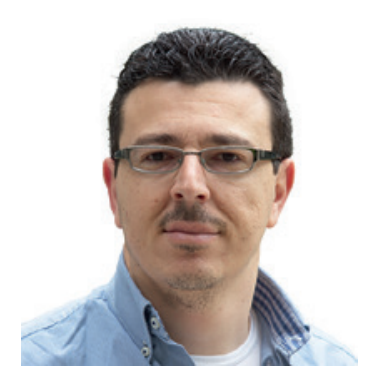

\title{
Effectiveness of eHealth and mHealth Interventions to Improve Physical Activity, Sedentary Behavior, and Nutrition in Healthy Subjects
}

\author{
Nikolaos Papaspanos
}

Thessaloniki, Greece

Abstract from Fiedler J, Eckert T, Wunsch K, Woll A: Key facets to build up eHealth and mHealth interventions to enhance physical activity, sedentary behavior and nutrition in healthy subjects - an umbrella review. BMC Public Health. 2020;20(1):1605.

\section{Keywords}

Exercise · Food and nutrition · Health behavior · Just-in-time adaptive intervention · Primary prevention · Psychological theory · Psychology social · Sedentary behavior - Telemedicine Umbrella review

\begin{abstract}
Background: Electronic (eHealth) and mobile (mHealth) health interventions can provide a large coverage, and are promising tools to change health behavior (i.e. physical activity, sedentary behavior and healthy eating). However, the determinants of intervention effectiveness in primary prevention has not been explored yet. Therefore, the objectives of this umbrella review were to evaluate intervention effectiveness, to explore the impact of pre-defined determinants of effectiveness (i.e. theoretical foundations, behavior change techniques, social contexts or just-intime adaptive interventions), and to provide recommendations for future research and practice in the field of primary prevention delivered via e/mHealth technology.
\end{abstract}

Methods: PubMed, Scopus, Web of Science and the Cochrane Library were searched for systematic reviews and meta-analyses (reviews) published between January 1990 and May 2020. Reviews reporting on e/mHealth behavior change interventions in physical activity, sedentary behavior and/or healthy eating for healthy subjects (i.e. subjects without physical or physiological morbidities which would influence the realization of behaviors targeted by the respective interventions) were included if they also investigated respective theoretical foundations, behavior change techniques, social contexts or just-in-time adaptive interventions. Included studies were ranked concerning their methodological quality and qualitatively synthesized.

Results: The systematic search revealed 11 systematic reviews and meta-analyses of moderate quality. The majority of original research studies within the reviews found $\mathrm{e} / \mathrm{mH}$ ealth interventions to be effective, but the results showed a high heterogeneity concerning assessment methods and outcomes, making them difficult to compare. Whereas theoretical foundation and behavior change techniques were suggested to be potential positive determinants of effective interventions, the impact of social context remains unclear. None of the reviews included just-in-time adaptive interventions.

Conclusion: Findings of this umbrella review support the use of e/mHealth to enhance physical activity and healthy eating and reduce sedentary behavior. The general lack of precise reporting and comparison of confounding variables in reviews and original research studies as well as the limited number of reviews for each health behavior constrains the generalization and interpretation of results. Further research is needed on study-level to investigate effects of versatile determinants of e/mHealth efficiency, using a theoretical foundation and additionally explore the impact of social contexts and more sophisticated approaches like just-in-time adaptive interventions.

Trial registration: The protocol for this umbrella review was a priori registered with PROSPERO: CRD42020147902.

(c) 2020 The Author(s) 


\section{Knowledge Transfer}

\section{Background}

Electronic (eHealth) and mobile (mHealth) tools can be used in interventions pertaining to physical activity, sedentary behavior, and healthy eating. The COVID-19 pandemic forced people to stay at home and rely on technology to communicate with each other and their therapist. This quarantine created certain situations that led to decreased physical activity and poorer dietary habits. According to Ammar et al. [1], physical activity significantly decreased during the COVID-19 lockdown. The days/week exercise rate decreased by $24 \%$, the minutes/day rate by $33.5 \%$, and the MET values for all physical activity by $38 \%$, while the number of hours per day spent sitting increased by $28.6 \%$. Furthermore, changes in eating behavior occurred during lockdown with a significant increase in unhealthy food consumption, binge eating, snacking between meals (including late-night snacks), and the number of main meals.

\section{Conclusion for Clinical Practice}

There are a lot of eHealth and mHealth tools that can be used in interventions pertaining to physical activity, sedentary behavior, and healthy eating. Their effectiveness, including in special populations, has been evaluated by many reviews. For example, Rollo et al. (2016) [7] examined if eHealth technologies can support nutrition and physical activity behaviors in diabetes self-management, while Grist et al. (2017) [4] evaluated mental health mobile apps for preadolescents and adolescents. Other reviews are aimed at the general population, such as that by Mateo et al. (2015) [5] which evaluated the use of mobile phone apps to promote weight loss and increase physical activity and Norman et al. (2007) [6] which evaluated eHealth intervention studies in adults and children targeting behavior change for physical activity, healthy eating, or both. Furthermore, Zhou et al. (2019) [9] created and validated an mHealth App Usability Questionnaire. All reviews, including that by Fiedler et al. (2020) [3], conclude that the findings for use of e/ $\mathrm{mHealth}$ tools to promote physical activity and healthy eating are promising.

There are some suggestions by the authors. Ernsting et al. (2017) [2] propose that app developers and researchers take into account the needs of older people and of those with low health literacy and chronic conditions, while Schmidt-Kraepelin et al. (2020) [8] suggest that gamification of mHealth apps may lead to better results. Further innovation, optimization, and rigorous research to maximize the potential of mHealth technology will move the field toward improved health care delivery and outcomes.

\section{Disclosure Statement}

I hereby declare that there are no conflicts of interest with regard to this commentary.

\section{References}

1 Ammar A, Brach M, Trabelsi K, et al.: Effects of COVID-19 Home Confinement on Eating Behavior and Physical Activity: Results of the ECLB-COVID19 International Online Survey. Nutrients. 2020;12(6):1583.

2 Ernsting C, Dombrowski US, Oedekoven M, et al.: Using Smartphones and Health Apps to Change and Manage Health Behaviors: A Population-Based Survey. J Med Internet Res. 2017;19(4):e101.

3 Fiedler J, Eckert T, Wunsch K, Woll A: Key effects to build up eHealth and mHealth interventions to enhance physical activity, sedentary behavior and nutrition in healthy subjects - an umbrella review. BMC Public Health. 2020;20(1):1605.

4 Grist R, Porter J, Stallard P: Mental Health Mobile Apps for Preadolescents and Adolescents: A Systematic Review. J Med Internet Res. 2017;19(5):e176.

5 Mateo GF, Granado-Font E, Ferré-Grau C, Montaña-Carreras X:Mobile Phone Apps to Promote Weight Loss and Increase Physical Activity: A Systematic Review and Meta-Analysis. J Med Internet Res. 2015;17(11):e253.

6 Norman GJ, Zabinski MF, Adams MA, et al.: A Review of eHealth Interventions for Physical Activity and Dietary Behavior Change. Am J Prev Med. 2007;33(4):336-345.

7 Rollo ME, Aguiar EJ, Williams LR, et al.: eHealth technologies to support nutrition and physical activity behaviors in diabetes self-management. Diabetes Metab Syndr Obes. 2016;9:381-390.

8 Schmidt-Kraepelin M, Toussaint AP, Thiebes S, et al.: Archetypes of Gamification: Analysis of mHealth Apps. JMIR Mhealth Uhealth. 2020;8(10):e19280.

9 Zhou L, Bao J, Setiawan IMA, et al.: The mHealth App Usability Questionnaire (MAUQ): Development and Validation Study. JMIR Mhealth Uhealth. 2019;7(4):e11500.

Correspondence to:

Nikolaos Papaspanos,nmpapaspanos @ yahoo.gr 